\title{
Editorial
}

\section{Acute Kidney Injury: A Modifiable Risk Factor for Cardiovascular Morbidity and Mortality}

\author{
Gaurav Alreja Jay L. Koyner \\ Section of Nephrology, Department of Medicine, University of Chicago, Chicago, III., USA
}

Acute kidney injury (AKI) occurs in a significant proportion of patients undergoing cardiovascular interventions and is associated with long-term morbidity and mortality in patients undergoing both non-cardiac surgery and cardiac surgery [1-4]. In a systemic review of 13 cohort studies, Coca et al. [5] identified AKI as an independent risk factor for the development of chronic kidney disease and its most severe form, the end-stage renal disease. In addition to these associations, longer duration of postoperative AKI has also been linked with increased long-term, all-cause mortality [4]. In this edition of the American Journal of Nephrology, Saratzis and colleagues, further strengthen these associations by demonstrating increased risk of cardiovascular morbidity and mortality in those with a prior history of post-operative AKI [6]. In their prospectively collected multicenter cohort of patients undergoing elective abdominal aortic aneurysm repair (endovascular or open), they concluded that after adjusting for known cardiovascular risk factors, AKI, regardless of surgical technique, was associated with increased long-term cardiovascular morbidity and mortality.

In their cohort of 947 subjects (median follow-up of 62 months), mortality rates of 11.8 and $8.3 \%$ were observed for patients undergoing endovascular and open repair re-

\section{KARGER 125\%}

(c) 2015 S. Karger AG, Basel

0250-8095/15/0424-0282\$39.50/0

E-Mail karger@karger.com

www.karger.com/ajn spectively. AKI, defined as at least a $\geq 26.4 \mu \mathrm{mol} / \mathrm{l}$ or 1.5 fold increase in serum creatinine from preoperative baseline, occurred in 188 (19.8\%) subjects. AKI was independently associated with cardiovascular events (composite cardiovascular end point: non-fatal myocardial infarction, non-fatal stroke, non-fatal peripheral vascular events, hospitalization due to heart failure, and death) with a hazard ratio $(95 \% \mathrm{CI}) 1.73(1.0-3.39) ; \mathrm{p}=0.03$. In addition to their own prospectively collected data, the authors also performed a meta-analysis that reiterated their findings in previously published retrospective studies of patients undergoing other cardiovascular interventions (e.g. primarily percutaneous coronary interventions). The meta-analysis, which included 12 separate studies in its final analyses, revealed an increased cardiovascular relative risk (RR) for patients undergoing PCI (RR (95\% CI) $1.76(1.45-2.12) ; \mathrm{p}<0.001, \mathrm{I}^{2}=50.6 \%$ at 1 year, $\mathrm{RR}$ (95\% CI) $1.34(1.10-1.63) ; \mathrm{p}=0.003, \mathrm{I}^{2}=33.9 \%$ at 2 years) and cardiac surgery (RR (95\% CI) $1.60(1.43-1.81), \mathrm{I}^{2}=$ $0 \%$ at 5 years).

COI: J.L.K. reports receiving funds for enrolling subjects in prospective observational biomarker studies and consulting fees from Astute Medical Inc.
Jay L. Koyner, MD

Section of Nephrology, Department of Medicine, University of Chicago 5841 South Maryland Ave, Suite S-506, MC5100 Chicago, IL 60637 (USA)

E-Mail jkoyner@uchicago.edu 
Importantly, the meta-analysis portion of this paper is inherently limited by publication bias and while the prospective observational study made every attempt to utilize clear and standardized adverse event definitions, these and the use of the death certificate data may be viewed as limitations of this study. Additionally, the prospective cohort, like those before it demonstrates only an association between AKI and adverse outcomes and not a causal effect. Thus, while we remain hopeful that interventions, which reduce the incidence or duration/severity of AKI will make a significant reduction in long-term cardiovascular morbidity and mortality, this remains unproven. To this end, while the Assessment, Serial Evaluation, and Subsequent Sequelae of Acute Kidney Injury (ASSESS AKI) study is being undertaken to help with this issue, it serves to identify and investigate the long-term consequences of $\mathrm{AKI}$ in high-risk patients who could be targeted for future interventional (prophylactic or therapeutic) trials [7]. Nonetheless, the current study from Saratzis and colleagues enhances our understanding of the depth of association between AKI and adverse outcomes, and should prompt further research on the pathophysiologic mechanisms explaining the increased risk of cardiovascular events.

Now that the link between AKI and increased risk of adverse cardiovascular outcomes has been further cemented by Saratzis et al., how should we intervene in those with AKI? Can we modify patient outcomes? Currently, the prevention and treatment of AKI in the setting of cardiovascular surgery/procedures revolves around a few central concepts including, consideration of the most appropriate procedure, attempts to minimize the time and usage of cardiopulmonary bypass, minimizing exposure to radio-contrast and other nephrotoxins and optimizing peri-procedure hemodynamics, perfusion pressures and oxygen delivery $[8,9]$. In addition to these currently available techniques, biomarkers of renal tubular injury and cell cycle arrest have recently demonstrated promise in determining those at risk of severe AKI and other adverse long-term outcomes. In those with early AKI, tissue inhibitor metalloproteinases- 2 and insulinlike growth factor-binding protein-7 measured early in the setting of critical illness was associated with a composite endpoint of 9-month all-cause mortality or the future need for renal replacement therapy [10]. Additionally, urinary interleukin-18, and kidney injury molecule-1 concentrations, in the immediate postoperative period (1-3 days) after cardiac surgery, was associated with increased 3-year all-cause mortality risk in patients with and without creatinine-based AKI [11]. Thus, it is easy to foresee using these new tools in concert with the afore- mentioned existing strategies in high risk patients to enroll subjects in future trials seeking to attenuate early AKI; this may in turn decrease long-term cardiovascular and all-cause morbidity and mortality.

In the past various pharmacological agents have been evaluated and many trials are currently ongoing to prevent AKI in patients undergoing elective or urgent cardiovascular procedures, the results however have been disappointing [12]. A recent study evaluated the nonpharmacological role of remote ischemic pre-conditioning in preventing $\mathrm{AKI}$ in patients undergoing cardiac surgery. In a randomized controlled multicenter trial of 240 high-risk subjects Zarbock et al. [2] showed a significantly reduced risk of AKI and the decreased need for renal replacement therapy in those receiving remote ischemic preconditioning. We are hopeful, that this paper, which also demonstrated the utility of biomarkers of AKI, is the first of many trials that will demonstrate a clinical benefit to those at high risk for AKI.

Over the last several decades, significant advances have been made in the modification of risk factors to prevent cardiovascular morbidity and mortality. Some estimates point to roughly one-half of all cardiovascular deaths in US adults aged 45-79 years being linked to major modifiable cardiovascular risk factors [13]. The work of Saratzis et al. [6] further links AKI and long-term cardiovascular morbidity and mortality. As nephrologists continue to develop tools that improve AKI risk stratification and diagnosis while performing studies to prevent and treat AKI, we must work toward a future where AKI is listed along with obesity and diabetes, as a modifiable cardiovascular risk factor that can be proactively treated to improve long-term outcomes.

References

1 Chertow GM, Levy EM, Hammermeister KE, Grover F, Daley J: Independent association between acute renal failure and mortality following cardiac surgery. Am J Med 1998;104: 343-348.

2 Zarbock A, et al: Effect of remote ischemic preconditioning on kidney injury among high-risk patients undergoing cardiac surgery: a randomized clinical trial. JAMA 2015; 313:2133-2141.

3 Chawla LS, et al: Association between AKI and long-term renal and cardiovascular outcomes in United States veterans. Clin J Am Soc Nephrol 2014;9:448-456.

4 Coca SG, King JT Jr, Rosenthal RA, Perkal MF, Parikh CR: The duration of postoperative acute kidney injury is an additional parameter predicting long-term survival in diabetic veterans. Kidney Int 2010;78:926-933. 
5 Coca SG, Singanamala S, Parikh CR: Chronic kidney disease after acute kidney injury: a systematic review and meta-analysis. Kidney Int 2012;81:442-448.

6 Saratzis AHS: Acute kidney injury is associated with long-term cardiovascular events after intervention. Am J Nephrol 2015;42: 285-294.

7 Go AS, et al: The assessment, serial evaluation, and subsequent sequelae of acute kidney injury (ASSESS-AKI) study: design and methods. BMC Nephrol 2010;11:22.
8 Brienza N, Giglio MT, Marucci M, Fiore T: Does perioperative hemodynamic optimization protect renal function in surgical patients? A meta-analytic study. Crit Care Med 2009;37:2079-2090.

9 Coleman MD, Shaefi S, Sladen RN: Preventing acute kidney injury after cardiac surgery. Curr Opin Anaesthesiol 2011;24:70-76.

10 Koyner JL, et al: Tissue inhibitor metalloproteinase-2 (TIMP-2) and IGF-binding protein-7 (IGFBP7) levels are associated with adverse long-term outcomes in patients with AKI. J Am Soc Nephrol 2015;26:1747-1754.
11 Coca SG, et al: Urinary biomarkers of AKI and mortality 3 years after cardiac surgery. J Am Soc Nephrol 2014;25:1063-1071.

12 Faubel S, et al: Ongoing clinical trials in AKI. Clin J Am Soc Nephrol 2012;7:861-873.

13 Patel SA, Winkel M, Ali MK, Narayan KM, Mehta NK: Cardiovascular mortality associated with 5 leading risk factors: national and state preventable fractions estimated from survey data. Ann Intern Med 2015;163:245253 